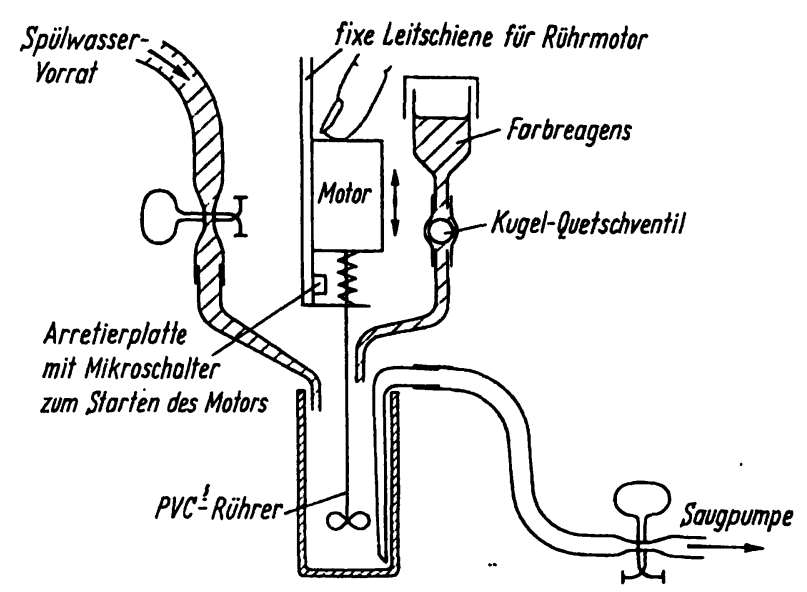

Abb. 2

(s. Abb. 2). - Zwischen den Messungen soll die Küvette mit Wasser gespült werden, damit nicht Reste von Farbreagens der vorhergehenden Probe in der neuen Probe die Eigenextinktion verfälschen. Ein rasches Arbeiten wird ermöglicht, wenn ein Reservoir mit Spülwasser über dem Photometer aufgestellt und andererseits eine Absaugküvette verwendet wird. Eine solche kann ohne großen Aufwand improvisiert werden (vgl. Abb. 2). Das Mischen von „Teepol“-Reagens, Serum und Farbreagens wird mittels Plastikrührer (nicbt Glas, wegen Zerkratzens der Küvette!) vorgenommen. Auch hier kann man sich ein paar Handgriffe ersparen, wenn das Rühren mechanisiert wird (vgl. Abb. 2). Ein kleiner Batteriemotor mit PVCRührer, der auf einer fixen Schiene vertikal verschiebbar ist, wird durch eine Feder so festgehalten, daß der Rührpropeller in Ruhe oberhalb des Flüssigkeitsniveaus der Küvette steht. Durch Fingerdruck wird die Feder komprimiert, der Rührer in die Küvette getaucht und gleichzeitig durch einen Mikroschalter am fixen Arretierplättchen der Stromkreis geschlossen. Zum Ablesen der Extinktion wird der Fingerdruck aufgehoben. Der Rührer steht still und hebt sich über den Lichtweg. Da der Propeller nur läuft, wenn er vollständig untergetaucht ist, bildet sich kein Schaum in der Lösung. Rührmotor, Farbreagensbürette und evtl. auch die Spülwasserflasche werden an einem Stativ neben dem Photometer befestigt.

Für wert volle Ratschläge und die Konstruktion der technischen Hilfsmittel bin ich Herrn J. Trachsel zu besonderem Dank verpflichtet.

\title{
Literatur
}

1. Ramsay, W. N. M., Advances Clin. Chem. 1, 1 (1958). - 2. Landers, J. W. und B. Zak, Amer. J. Clin. Path. 29, 590 (1958). - 3. Sanford, R., J. Clin. Path., London 16, 174 (1963).

\section{Zur Bestimmung körpereigener Amine in biologischen Substraten}

\section{Mitteilung: Bestimmung von $\mathbf{N}, \mathbf{N}-$ Dimethyltryptamin in Blut und Harn}

\author{
Von H. Gross und Fr. Franzen \\ Aus der Medizinischen Klinik der Universität Köln (Direktor: Prof. Dr. Dr. Dr. H. W. Knipping)
}

(Der Schriftleitung zugegangen am 1. Dezember 1964)

\begin{abstract}
Es wird ein fluorimetrisches Verfahren zur quantitativen Bestimmung des N,N-Dimethyltryptamins in Blut und Harn beschrieben. Während die in biologischen Substraten vorkommenden Homologen durch Umsetzung mit Schwefelkohlenstoff und andere ebenfalls in den gleichen Wellenbereichen fluoreszierende Verbindungen durch den Trennungsgang eliminiert werden, können Indol, Skatol und Indol-3-aldehyd ab einer bestimmten Konzentration das Analysenergebnis im Sinne eines Plusfehlers beeinträchtigen. Im Blut Gesunder fanden sich 0,0398 $\pm 0,006 \mu \mathrm{g}$ Dimethyltryptamin $/ \mathrm{m} l$, im Harn $42,98 \pm 8,59 \mu \mathrm{g} / 24$ Stdn.
\end{abstract}

A fluorimetric method is described for the quantitative determination of $\mathrm{N}, \mathrm{N}$-dimethyltryptamine in blood and urine. Homologues, which fluoresce at the same wavelengths and are present in biological substrates, can be eliminated by reaction with carbon disulphide or by extraction. Above a certain concentration, indole, skatole, and indole-3-aldehyde cause a positive error in the analytical results. The blood of healthy patients contained 0.398 $\pm 0.006 \mu \mathrm{g} / \mathrm{ml}$, urine $42.98 \pm 8.59 \mu \mathrm{g} / 24 \mathrm{hrs}$.

Für die Bestimmung von N,N-Dimethyltryptamin liegen bisher nur biologische Verfahren (1-3) vor. Sie erlauben unter Auswertung des pharmakologischen Effektes dieser Verbindung auf isolierte Organe - z. B. Rattenuterus, Molluskenherz - den Nachweis von $10^{-8} \mathrm{~g} / \mathrm{m} l$, wurden jedoch lediglich auf reine Aminlösungen, nicht auf biologische Substrate angewandt.

N,N-Dimethyltryptamin ist zur Eigenfluoreszenz befähigt. Nach unseren Untersuchungen besitzt es wie die übrigen Indolaethylamine (Tryptamin (4), N-Methyl- 
tryptamin) bei $\mathrm{pH}=8,3$ eine Aktivierungswellenlänge von $\lambda=275 \mathrm{~m} \mu$ und eine Fluoreszenzwellenlänge von $\left.\lambda=355 \mathrm{~m} \mu^{1}\right)$. Voraussetzung einer spektrophotofluorimetrischen Bestimmung des N,N-Dimethyltryptamins in biologischen Substraten wäre demnach die Eliminierung etwa vorhandener Homologen (Tryptamin, NMethyltryptamin) sowie die Proportionalität von Konzentration und Fluoreszenzintensität.

\section{Versuche}

Trennung der Indolaetbylamine durch Umsetzung mit $\mathrm{CS}_{2}$

Zur Trennung des Dimethyltryptamins von seinen Homologen Tryptamin und Methyltryptamin empfahl sich die in der 1. Mitteilung (6) für die 5-Hydroxindolaethylamine untersuchte Umsetzung mit Schwefelkohlenstoff. - Zur Klärung der Reaktion der Indolaethylamine mit $\mathrm{CS}_{2}$ wurden wäßrige, mit Natronlauge alkalisierte Lösungen von Tryptamin, Methyltryptamin bzw. Dimethyltryptamin mit Benzol extrahiert. Benzol extrahiert Indolaethylamine aus alkalischer Lösung rollständig; sein Lösungsvermögen für Verbindungen mit phenolischen Hydroxylgruppen - z. B. Serotonin, Methylserotonin, Bufotenin, Noradrenalin, Adrenalin, Oestradiol-[3,17- $\beta]$ - ist dagegen sehr gering. Die organische Phase wurde mit Schwefelkohlenstoff versetzt und geschüttelt, die gebildeten N-substituierten Dithiokarbaminsäuren mit Natronlauge extrahiert. Aus einem aliquoten Teil der organischen Phase wurde das Amin mittels Salzsäure extrahiert und in der wäßrigen Lösung fluorimetrisch bestimmt. Dabei wurde vorgelegtes $\mathrm{Di}$ methyltryptamin zu 99\%, Methyltryptamin zu 72\% und Tryptamin zu 63\% wiedergefunden. Das besagt, daß sich Dimethyltryptamin nicht, Methyltryptamin und Tryptamin unter den gewählten Versuchsbedingungen nur unvollständig mit $\mathrm{CS}_{2}$ umsetzt. $\mathrm{Nahm}$ man' die Amine dagegen in Essigsäureaethylester auf und führte in diesem die Reaktion mit Schwefelkohlenstoff durch, so wurde vorgelegtes Dimethyltryptamin zu $98 \%$, Methyltryptamin zu 6\% und Tryptamin zu 4\% wiedergefunden. Die in Benzol bzw. Essigsäureäthylester festgestellten unterschiedlichen Umsetzungsraten zwischen Methyltryptamin bzw. Tryptamin und Schwefelkohlenstoff lassen an eine Reaktionsbegünstigung durch den Essigsäureäthylester denken. Diese könnte darin zu sehen sein, daß der Essigsäureäthylester beim Schütteln der natronalkalischen wäßrigen Aminlösung auch Spuren Hydroxylionen löst, die ihrerseits durch einen polarisierenden Effekt die Umsetzung des $\mathrm{CS}_{2}$ mit Tryptamin und Methyltryptamin aktivieren. Sollte diese Annahme zutreffen, müßte auch in Benzol unter Zusatz einer polarisierenden Substanz eine bessere Umsetzung der Reaktionspartner zu erreichen sein. Wie aus Tabelle 1 hervorgeht, wurde durch-den Zusatz von $0,1 \mathrm{~m} /$ Dimethylsulfoxyd eine nahezu vollständige, durch $0,1 \mathrm{~m} l$ Pyridin eine quantitative Umsetzung von Trypt-

1) „Aminco-Bowman“-Spektrophotofluorimeter mit einer Photomultiplier-Röhre „1 P 28“; die angegebenen Wellenlängen sind nicht korrigiert. amin und Methyltryptamin mit Schwefelkohlenstoff erzielt. Dimethyltryptamin setzte sich unter diesen Versuchsbedingungen mit $\mathrm{CS}_{2}$ nicht um.

Die Möglichkeit einer Trennung des Dimethyltryptamins von seinen Homologen mittels der Schwefelkohlenstoffreaktion wurde auch unter biologischen Bedingungen, $d$. h. an menschlichem Blut und Urin in der Weise überprüft, daß diesen Substraten bekannte Mengen Indolaethylamine zugesetzt wurden. Wie aus $\mathrm{Ta}$ belle 2 hervorgeht, wurden Tryptamin und Methyl-

Tab. 1

Einfluß eines Dimethylsulfoxyd- bzw. Pyridin-Zusatzes auf die Umsetzung der Indolaethylamine mit Schwefelkohlenstoff in Benzol

\begin{tabular}{lccccccc}
\hline \multirow{2}{*}{ Zusatz } & \multicolumn{2}{c}{$\begin{array}{c}\text { Dimethyl- } \\
\text { tryptamin }\end{array}$} & \multicolumn{2}{c}{$\begin{array}{c}\text { Methyl- } \\
\text { tryptamin }\end{array}$} & \multicolumn{2}{c}{ Tryptamin } \\
& $\begin{array}{c}\text { Ge- } \\
\text { geben }\end{array}$ & Ge- & Ge- & Ge- & Ge- & Ge- \\
& $\mu \mathrm{g} / \mathrm{m} l$ & $\%$ & $\mu \mathrm{g} / \mathrm{m} l$ & $\%$ & $\mu \mathrm{g} / \mathrm{m} l$ & $\%$ \\
& 0,05 & 99 & 0,05 & 11 & 0,05 & 7 \\
& 0,10 & 97 & 0,10 & 9 & 0,10 & 7 \\
& 0,20 & 97 & 0,20 & 9 & 0,20 & 6 \\
$0,1 \mathrm{~m} l$ & 0,30 & 98 & 0,30 & 6 & 0,30 & 5 \\
Dimethylsulfoxyd & 0,05 & 98 & 0,5 & 0 & 0,5 & 0 \\
& 0,10 & 97 & 1,0 & 0 & 1,0 & 0 \\
& 0,20 & 98 & 1,5 & 0 & 2,0 & 0 \\
$0,1 \mathrm{~m} /$ Pyridin & 0,30 & 98 & 2,0 & 0 & 3,0 & 0 \\
\hline
\end{tabular}

Tab. 2

Wiederfindung von Dimethyltryptamin-, Methyltryp:amin- und Tryptamin-Zusä!zen in Harn und Blut

\begin{tabular}{|c|c|c|c|c|c|c|c|}
\hline & \multirow{3}{*}{$\begin{array}{l}\text { Dimethỳl- } \\
\text { tryptamin- } \\
\text { Eigengehalt } \\
\mu \mathrm{g} / \mathrm{m} /\end{array}$} & \multicolumn{2}{|c|}{$\begin{array}{l}\text { Dimethyl- } \\
\text { tryptamin }\end{array}$} & \multicolumn{2}{|c|}{$\begin{array}{l}\text { Methyl- } \\
\text { tryptamin }\end{array}$} & \multicolumn{2}{|c|}{ Tryptamin } \\
\hline & & \multirow{2}{*}{$\begin{array}{l}\text { Ge- } \\
\text { geben } \\
\mu \mathrm{g} / \mathrm{m} l\end{array}$} & \multirow{2}{*}{$\begin{array}{c}\text { Ge- } \\
\text { funden } \\
\%\end{array}$} & \multirow{2}{*}{$\begin{array}{c}\text { Ge- } \\
\text { geben } \\
\mu \mathrm{g} / \mathrm{m} l\end{array}$} & \multirow{2}{*}{$\begin{array}{l}\text { Ge- } \\
\text { funden } \\
\%\end{array}$} & \multirow{2}{*}{$\begin{array}{l}\text { Ge- } \\
\text { geben } \mathrm{f} \\
\mu \mathrm{g} / \mathrm{ml}\end{array}$} & \multirow{2}{*}{$\begin{array}{l}\text { Ge- } \\
\text { Eunden } \\
\%\end{array}$} \\
\hline & & & & & & & \\
\hline \multirow{13}{*}{ Harn } & 0,018 & 0,020 & 95 & & & \multirow{13}{*}{$\begin{array}{l}0,047 \\
0,140 \\
1,40 \\
3,30\end{array}$} & \\
\hline & 0,018 & 0,033 & 96 & & & & \\
\hline & 0,018 & 0,040 & 97 & & & & \\
\hline & 0,018 & 0,047 & 100,7 & & & & \\
\hline & 0,018 & 0,060 & 95,5 & & & & \\
\hline & 0,024 & & & 0,33 & 0 & & \\
\hline & 0,024 & & & 0,67 & 0 & & \\
\hline & 0,039 & & & 1,40 & 0 & & \\
\hline & 0,039 & & & 2,00 & 0 & & \\
\hline & 0,033 & & & & & & 0 \\
\hline & 0,033 & & & & & & 0 \\
\hline & 0,033 & & & & & & 0 \\
\hline & 0,018 & & & & & & 0 \\
\hline \multirow{14}{*}{ Blut } & 0,010 & 0,05 & 88 & \multirow{14}{*}{$\begin{array}{l}0,2 \\
0,5 \\
1,0 \\
1,5\end{array}$} & & \multirow{14}{*}{$\begin{array}{l}0,5 \\
1,0 \\
1,5 \\
2,0 \\
2,5\end{array}$} & \\
\hline & 0,010 & 0,10 & 89,5 & & & & \\
\hline & 0,015 & 0,10 & 81 & & & & \\
\hline & 0,021 & 0,50 & 81 & & & & \\
\hline & 0,021 & 0,50 & 79 . & & & & \\
\hline & 0,009 & & & & 0 & & \\
\hline & 0,009 & & & & 0 & & \\
\hline & 0,009 & & & & 0 & & \\
\hline & 0,015 & & & & 0 & & \\
\hline & 0,021 & & & & & & 0 \\
\hline & 0,021 & & & & & & 0 \\
\hline & 0,021 & & & & & & 0 \\
\hline & 0,015 & & & & & & 0 \\
\hline & 0,015 & & & & & & 0. \\
\hline
\end{tabular}


tryptamin aus dem Reaktionsgemisch vollständig eliminiert, Dimethyltryptamin - nach Abzug des vorher bestimmten Substrateigengehaltes - im Urin zu 97\%, im Blut $z u \sim 80 \%$ wiedergefunden.

\section{Eliminierung anderer fluoreszierender Verbindungen}

Nachdem die Homologen Methyltryptamin und Tryptamin durch Reaktion mit $\mathrm{CS}_{2}$ aus biologischen Substraten vollständig eliminiert werden können, erhebt sich im Hinblick auf eine fluorimetrische Bestimmung des im Analysengang verbleibenden Dimethyltryptamins die Frage nach dem Störeinfluß anderer fluoreszierender Substanzen. Hierbei ist zunächst eine Gruppe von Verbindungen zu erwähnen, die zwar in den gleichen bzw. benachbarten Wellenbereichen fluoreszieren, jedoch durch den Trennungsgang (z. B. die Benzolextraktion oder die Umsetzung mit $\mathrm{CS}_{2}$ ) entfernt werden. Dies sind Serotonin, Methylserotonin, Bufotenin, Indikan, Noradrenalin, Adrenalin, Adenin, Allantoin, Oestradiol$[3,17-\beta]$ und Vitamin E. Als zweite Gruppe sind Verbindungen zu berücksichtigen, die in den gleichen bzw. angrenzenden Wellenbereichen fluoreszieren, durch den bisher entwickelten Trennungsgang jedoch nicbt eliminiert werden und oberhalb einer bestimmten Konzentration (Indol 1,12 $\mu \mathrm{g} / \mathrm{m} l$, Skatol $0,6 \mu \mathrm{g} / \mathrm{m}$, Indol-3aldehyd $1,44 \mu \mathrm{g} / \mathrm{m} l$, Gramin $.0,001 \mu \mathrm{g} / \mathrm{ml}$ ) die fluorimetrische Dimethyltryptamin-Bestimmung im Sinne eines Plusfehlers beeinträchtigen. Dieser beträgt 3\% für die angegebenen Mengen der in Klammern aufgeführten Verbindungen. Abgesehen von der Frage nach dem Vorkommen dieser Verbindungen in menschlichem Blut und Harn, sind eigene Untersuchungen zu deren Abtrennung im Gange. Eine Eliminierung des - in tierischen und menschlichen Substraten bisher noch nicht nachgewiesenen - Gramins (= $\beta$-Indoxyl-methyl-dimethylamin) könnte $z$. B. in der Weise erfolgen, $\mathrm{da} ß$ dieses mit wäßriger Polysulfidlösung versetzt und im Wasserbad auf $90^{\circ}$ erhitzt wird; nach 1-stündiger Dauer hat Gramin seine Fluoreszenz vollkommen verloren, während in gleicher Weise behandeltes Dimethyltryptamin einen Konzentrationsverlust von $6-9 \%$ exleidet.

\section{Abbängigkeit der Fluoreszenzintensität von der Dimetbyl- tryptaniin-Konzentration}

Zur Prüfung der linearen Abhängigkeit der Fluoreszenzintensität von der Konzentration wurde eine Verdünnungsreihe von $0,015-0,15 \mu \mathrm{g}$ Dimethyltryptamin $/ \mathrm{m} l$ nach der für die Aminbestimmung im Harn nachfolgend näher besprochenen Methode aufgearbeitet. Die graphische Darstellung der Bezugsgrößen (Abb. 1) ergab eine durch den Nullpunkt führende Gerade und damit eine lineare Abhängigkeit der Fluoreszenzintensität von der Dimethyltryptamin-Konzentration.

\section{Auswertung}

Die Auswertung der fluorimetrischen Meßdaten erfolgt entweder mit Hilfe einer Eichkurve oder unter Verwendung einer Verfahrenskonstanten (5) oder durch

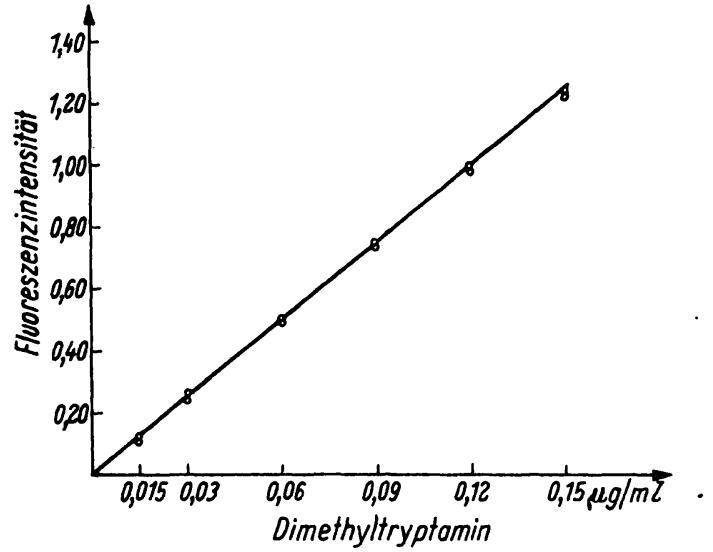

Abb. 1

Abhängigkeit der Fluoreszenzintensität von der Dimethyltryptamin-Konzentration

Tab. 3

Verfahrenskonstante und Standardabiveichung der Dimethyltryptaminbestimmung in Blut und Harn

Blut Harn

\begin{tabular}{cccc}
$\begin{array}{c}b=\mu \mathrm{g} \text { Dimethyl- } \\
\text { tryptamin } / \mathrm{m} l\end{array}$ & Meßwert & $\begin{array}{c}\text { wahrer } \\
\text { tryptamin/ml }\end{array}$ & $\begin{array}{c}W=\text { wahrer } \\
\text { Meßwert }\end{array}$ \\
\hline 0,50 & 0,40 & 0,150 & 1,2180 \\
0,50 & 0,42 & 0,150 & 1,2299 \\
0,50 & 0,39 & 0,150 & 1,2184 \\
0,50 & 0,39 & 0,150 & 1,2300 \\
0,40 & 0,34 & 0,120 & 0,9900 \\
0,40 & 0,30 & 0,120 & 0,9949 \\
0,40 & 0,32 & 0,120 & 1,0170 \\
0,40 & 0,32 & 0,120 & 0,9850 \\
0,30 & 0,24 & 0,090 & 0,7430 \\
0,30 & 0,25 & 0,090 & 0,7476 \\
0,30 & 0,23 & 0,090 & 0,7399 \\
0,30 & 0,24 & 0,090 & 0,7378 \\
0,20 & 0,16 & 0,060 & 0,5005 \\
0,20 & 0,17 & 0,060 & 0,5064 \\
0,20 & 0,14 & 0,060 & 0,4995 \\
0,20 & 0,16 & 0,060 & 0,4995 \\
0,10 & 0,10 & 0,030 & 0,2450 \\
0,10 & 0,09 & 0,030 & 0,2501 \\
0,10 & 0,08 & 0,030 & 0,2633 \\
0,10 & 0,09 & 0,030 & 0,2450 \\
0,05 & 0,04 & 0,015 & 0,1252 \\
0,05 & 0,04 & 0,015 & 0,1223 \\
0,05 & 0,03 & 0,015 & 0,1125 \\
0,06 & 0,06 & 0,015 & 0,1125 \\
\hline
\end{tabular}

Verfahrenskonstante:

$$
[\omega]=\frac{\Sigma b W}{\Sigma W^{2}} \quad \mu g / \mathrm{m} l
$$

Standardabweichung $s_{k}$ :

$$
\begin{aligned}
& s_{k}= \pm \sqrt{\frac{1}{f} \Sigma \nu_{k}^{2}} \quad \mu g / \mathrm{ml} \\
& \Sigma \nu_{k}^{2}=\Sigma b^{2}-[\omega] \Sigma b \mathrm{~W}
\end{aligned}
$$

\begin{tabular}{lll}
\hline & Blut & Harn \\
\hline$\Sigma b W$ & 1,7705 & 1,6376 \\
$\Sigma W^{2}$ & 1,4216 & 13,485 \\
{$[\omega]$} & 1,24542 & 0,121439 \\
$f=n-1$ & 23 & 23 \\
$\Sigma b^{2}$ & 2,210 & 0,1989 \\
$s k$ & 0,0147 & 0,0012 \\
\hline
\end{tabular}


Bezugnahme auf Standardwerte (6). Die streng methodeund gerätegebundene Verfahrenskonstante beträgt gemäß linearer Ausgleichsrechnung für die mit dem „Amico-Bowman"-Spektrophotofluorimeter durchgeführte Dimethyltryptamin-Bestimmung im Harn $0,12 \mu \mathrm{g}$ Amin $/ \mathrm{ml}\left( \pm \mathrm{s}_{\mathrm{k}}=0,001 \mu \mathrm{g} / \mathrm{m} l\right)$, für die Bestimmung im Blut $1,24 \mu \mathrm{g} \mathrm{Amin} / \mathrm{m} l\left( \pm \mathrm{s}_{\mathrm{k}}=0,014 \mu \mathrm{g} / \mathrm{m} l\right)$. Die untere Bestimmungsgrenze (vgl. 5) beträgt $0,005 \mu \mathrm{g} / \mathrm{ml}$, die untere Erfassungsgrenze $0,001 \mu \mathrm{g} / \mathrm{m} l$. Liegt ein Analysenergebnis unterhalb der vorerwähnten Bestimmungsgrenze, empfiehlt sich die Durchführung einer Zweitanalyse unter Ansatz eines der Probenkonzentration angepaßten Standardwertes.

Bestimmung des Dimethyltryptamins im Harn $20 \mathrm{~m} l$ des unter Vorlage von $20 \mathrm{~m} / 3-n-\mathrm{HCl}$ gesammelten 24-Stdn.Harns werden nach Zugabe von $1 \mathrm{~m} l$ kalt gesättigter Titriplex-IIILösung mit 33-proz. Natronlauge auf $\mathrm{pH}=11$ eingestellt, mit $5 \mathrm{~g}$ Natriumchlorid versetzt (Aussalzeffekt) und zur Extraktion der Indolaethylamine mit $20 \mathrm{~m} l$ Benzol 15 Minuten geschüttelt. Nach Zentrifugieren werden $15 \mathrm{ml}$ der benzolischen Phase abpipettiert, zur Bildung der N-substituierten Dithiokarbaminsäuren mit $1 \mathrm{~m} l$ Schwefelkohlenstoff sowie $0,1 \mathrm{~m} l$ Pyridin als Reaktionsvermittler versetzt und 15 Minuten geschüttelt. Die Entfernung der Dithiokarbaminsäuren erfolgt durch zweimaliges Schütteln der organischen Phase mit je $5 \mathrm{ml}$ einer NaCl-gesättigten 0,1-n-Natronlauge. Zur Überführung des Dimethyltryptamins in eine wäßrige Lösung werden $10 \mathrm{ml}$ der benzolischen Phase mit $1 \mathrm{~m} / 0,1-n-\mathrm{HCl} 15 \mathrm{Min}$. geschüttelt, zentrifugiert und die organische Phase verworfen. Zur Bestimmung des Amins werden $0,5 \mathrm{ml}$ des salzsauren Extrakts mit $1 \mathrm{ml}$ Boratpuffer ${ }^{1}$ ) versetzt und bei der

1) $1,7 \mathrm{~g}$ Borsäure p. a. in $200 \mathrm{~m} /$ bidest. Wasser gelöst, die Lösung mit $0,1-n-\mathrm{NaOH}$ (ca. $174 \mathrm{ml}$ ) auf $\mathrm{pH}=11$ eingestellt.
Aktivierungswellenlänge $\lambda=275 \mathrm{~m} \mu$ sowie der Fluoreszenzwellenlänge $\lambda=355 \mathrm{~m} \mu$ fluorometriert.

Bestimmung des Dimethyltryptamins im Blut $2 \mathrm{~m} l$ Blut werden mit $4,5 \mathrm{~m} l$ bidestilliertem Wasser haemolysiert, zur Enteiweißung mit $0,5 \mathrm{ml} 60$-proz. Perchlorsäure $15 \mathrm{Min}$. geschüttelt und zur schnellen und vollständigen Sedimentation des ausgeflockten Eiweißes bei $4000 \mathrm{U} / \mathrm{Min}$. zentrifugiert. Von der klaren, stark sauren Lösung werden $4 \mathrm{ml}$ abpipettiert, mit 33-proz. $\mathrm{NaOH}$ auf $\mathrm{pH}=11$ eingestellt, und die Indolaethylamine nach Zugabe von $2 \mathrm{~g} \mathrm{NaCl}$ (Aussalzeffekt) mit $20 \mathrm{ml}$ Benzol extrahiert. Der weitere Analysengang entspricht dem der Bestimmung des Amins im Harn.

\section{Diskussion}

Das besprochene Verfahren erlaubt in $2 \mathrm{ml}$ Blut bzw. $20 \mathrm{ml} \mathrm{Harn} \mathrm{-} \mathrm{in} \mathrm{etwa} 11 / 2$ Stdn. - eine quantitative Bestimmung des N,N-Dimethyltryptamins in biologischen Substraten. Die untere Erfassungsgrenze liegt bei $0,001 \mu \mathrm{g} \mathrm{Amin} / \mathrm{m} l$, die untere Bestimmungsgrenze bei $0,005 \mu \mathrm{g} / \mathrm{m} l$, die Fehlerbreite des Verfahrens $\pm 0,003 \mu \mathrm{g}$ $\mathrm{Amin} / \mathrm{ml}$. Während zahlreiche andere in den gleichen Wellenbereichen fluoreszierende Verbindungen durch den Trennungsgang eliminiert werden, können Indol, Skatol und Indol-3-aldehyd - sofern sie in dem zu untersuchenden biologischen Substrat vorliegen - ab einer bestimmten Konzentration das Analysenergebnis im Sinne eines Plusfehlers beeinträchtigen. - An je 50 Gesunden fanden sich im Blut 0,0398 $\pm 0,006 \mu \mathrm{g}$ Dimethyltryptamin $/ \mathrm{m} l$, im Harn $42,98 \pm 8,59 \mu \mathrm{g} / 24$ Stdn.

\title{
Literatur
}

1. Chen, K. K. und A. L. Chen, J. Amer. pharmac. Assoc., Sci. Edit. 22, 813 (1933). - 2. Greenberg, M. J., Brit. J. Pharmacol. Chemotherapy 15, 365 und 375 (1960). - 3. Bertaccini, G. und P. Zambonr, Arch. int. Pharmacodynam. Thérap. 133, 138 (1961). - 4. Sjoerdsma, A., J. A. Oates, P. Zaltzman und
S. UdENFrIEND, J. Pharmacol. exper. Therap. 126, 217 (1959). 5. Gotrschalk, G., Statistik in der quantitativen chemischen Analyse. Ferdinand Enke-Verlag, Stuttgart (1962). - 6. Gross, H. und Fr. Franzen, Biochem. Z. 340, 403 (1964).
Priv.-Doz. Dr. med. Fr. Franzen 5 Köln-Marienburg, Ulmenallee 3

\section{KURZMITTEILUNG}

\section{Eine Routinemethode zur Bestimmung von Serumeisen}

\author{
Von E. Deggau, F. Kröhnke, K. E. Schnalke, HJ. Staudinger und W. Weis \\ Aus dem Physiologisch-Chemischen Institut (Direktor: Prof. Dr. Hj. Staudinger) und dem Institut für Organische Chemie \\ (Direktor: Prof. Dr. F. Kröbnke) der Justus Liebig-Universität Giessen
}

(Der Schriftleitung zugegangen am 20. März 1965)

Alle im klinisch-chemischen Laboratorium routinemäßig durchgeführten Eisenbestimmungen beruhen auf der Bildung farbiger Komplexe des zweiwertigen Eisens mit 2,2'-Dipyridyl oder 1,10-Phenanthrolin und seinen Derivaten (1). Auch $2,2^{\prime}, 2^{\prime \prime}$-Tripyridyl wurde schon zur empfindlichen Eisenbestimmung empfohlen (2,3); dieses Reagens ist jedoch schwer zugänglich. Im' 2,6-Di[pyridyl-(2)]-4-[p-methoxy-phenyl]-pyridin (I) wurde ein jetzt leicht zugänglicher und spezifischer Komplexbildner gefunden, der mit $\varepsilon=26900$ eine der größten 